\title{
The relationship between disability and clinical outcomes in maintenance dialysis patients
}

\author{
Seok Hui Kang ${ }^{1}$, Jun Young Do ${ }^{1}$, Jun Chul Kim ${ }^{2}$ \\ ${ }^{1}$ Division of Nephrology, Department of Internal Medicine, Yeungnam University College of Medicine, Daegu, Korea \\ ${ }^{2}$ Division of Nephrology, Department of Internal Medicine, CHA Gumi Medical Center, CHA University, Gumi, Korea
}

Received: May 15, 2020

Revised: September 10, 2020

Accepted: September 11, 2020

Corresponding author:

Jun Chul Kim, MD, PhD

Division of Nephrology, Department of Internal Medicine, CHA Gumi

Medical Center, CHA University, 12

Sinsi-ro 10-gil, Gumi 39295, Korea

Tel: +82-54-450-9550

Fax: +82-54-450-9790

E-mail:truedoc1@hanmail.net

\begin{abstract}
Background: Dialysis patients are prone to having disabilities. We aimed to evaluate the association between disability and various clinical outcomes in Korean dialysis patients.

Methods: This study consisted of 1,615 dialysis patients from 27 centers. We evaluated disability by using four questions on the activities of daily living (ADLs) concerning whether help was needed for feeding, dressing/undressing, getting in/out of bed, or taking a bath/shower. We divided the patients into three groups: no disability (Non-D, none of the four ADL domains required help; $n=1,312$ ), mild disability (Mild-D, one ADL domain required some/full help; $n=163$ ), or moderate to severe disability (MS-D, two or more $A D L$ domains required some/full help; $n=140$ ). We evaluated falls, frailty, health-related quality of life (HROoL), mortality, and hospitalization. Results: The numbers of participants with a fall during the last 1 year were 199 (15.2\%), 42 $(25.8 \%)$, and $44(31.4 \%)$ in the Non-D, Mild-D, and MS-D groups, respectively $(p<0.001)$. The numbers of participants with frailty in the Non-D, Mild-D, and MS-D groups were 381 (29.0\%), $84(51.5 \%)$, and $93(66.4 \%)$, respectively $(p<0.001)$. In both univariate and multivariate analyses, the physical component scale and mental component scale scores decreased as the grade of disability increased ( $p<0.001$ for both scores). Hospitalization-free survival rate at 500 days was $64.2 \%, 56.7 \%$, and $51.1 \%$ in the Non-D, Mild-D, and MS-D, respectively ( $p=0.001$ for trend). Patient survival rate at 500 days was $95.3 \%, 89.5 \%$, and $92.3 \%$ in the Non-D, Mild-D, and MS-D, respectively ( $p=0.005$ for trend).

Conclusion: Disability was associated with falls, frailty, HRQoL scales, and survival trends in Korean dialysis patients.
\end{abstract}

Keywords: Dialysis; Disabled persons; Quality of life; Treatment outcome

\section{Introduction}

Patients with end-stage renal disease require renal replacement therapy, which includes dialysis and kidney transplantation. Although kidney transplantation is the best option for renal replacement therapies, most patients undergo dialysis. In the United States, 124,114 patients were diagnosed with incident end-stage renal disease; among them, $87.8 \%$ underwent hemodialysis (HD) and $9.6 \%$ underwent peritoneal dialysis (PD) [1]. Dialysis patients have poor survival and clinical outcomes compared with the general population. Many researchers have tried to identify risk factors for poor prognosis in dialysis patients. However, the prognosis remains poor, and studies about new risk factors are ongoing.

According to the World Health Organization (WHO), disability can be defined as an umbrella term, which includes impairment, activity limitation, and participation restrictions [2]. Disability is associated with poor clinical outcomes in the general population, due to inaccessibility to health care services, and vulnerability to

Copyright (C) 2021 Yeungnam University College of Medicine

This is an Open Access article distributed under the terms of the Creative Commons Attribution Non-Commercial License (http://creativecommons.org/licenses/by-nc/4.0/) which permits unrestricted non-commercial use, distribution, and reproduction in any medium, provided the original work is properly cited. 
infection. Many comorbid conditions lead to the development of disability, and end-stage renal disease is associated with many comorbid conditions such as diabetes mellitus (DM), hypertension, and uremia. Therefore, dialysis patients are prone to having disabilities. Jassal et al. [3] analyzed dialysis outcomes and practice patterns in a cohort of 7,226 patients from 12 countries, and showed that disability is associated with low health-related quality of life (HRQoL) and poor survival. Additionally, Yazawa et al. [4] used a Japanese national registry of 7,664 $\mathrm{HD}$ patients, and showed an association between disability and mortality. However, previous studies have included limited ethnicities and only focused on survival or HRQoL. Regional and national differences must be considered. Hence, we aimed to evaluate the association between disability and various clinical outcomes in Korean dialysis patients.

\section{Materials and methods}

This study was approved by the Institutional Review Board (IRB) of Yeungnam University Hospital (IRB No: 2016-06-022). Informed consent was obtained from all individual participants included in the study.

\section{Study population}

We used data from a previous study [5]. Briefly, a total of 2,737 study participants underwent maintenance dialysis and were enrolled from 27 dialysis centers between July and December 2012. Patients were excluded for the following reasons: age $<20$ years $(\mathrm{n}=12)$, dialysis vintage $<6$ months $(\mathrm{n}=164)$, a history of hospitalization in the prior 3 months for any other than an $\mathrm{HD}$ vascular access $(n=351)$, inability to walk with or without an assistive device $(\mathrm{n}=79)$, inability to communicate with the interviewer $(n=149)$, refusal to provide informed consent $(n=254)$, or insufficient data $(n=113)$. Finally, a total of 1,615 patients were recruited into our study (Fig. 1; 1,249 and 366 patients underwent HD and PD, respectively). The participants were recruited between

Patients assessed for eligibility $(n=2,737)$

Exclusion criteria

12 Excluded due to age $<20 \mathrm{yr}$

164 Excluded due to dialysis vintage $<6$ mo

$351 \mathrm{Had}$ history of hospitalization in the prior $3 \mathrm{mo}$

79 Excluded due to inability to ambulate

254 Refused to consent

149 Excluded due to inability to communicate

$113 \mathrm{Had}$ missing data

1,615 Patients included in the analysis

Fig. 1. Study flow chart.
July and December 2012; the follow-up ended in March 2014. During the follow-up period, transfer to other hospitals, change of dialysis modality, kidney transplantation, or follow-up loss was considered as censored data.

\section{Study variables}

In our study, all variables except patient survival and hospitalization rates were defined using findings at the enrollment. Our study examined age, sex, body mass index (BMI $\left.\mathrm{kg} / \mathrm{m}^{2}\right), \mathrm{DM}$, coronary artery disease $(\mathrm{CAD})$, cerebrovascular disease $(\mathrm{CVD})$, dialysis vintage, and education level, as well as hemoglobin $(\mathrm{mg} / \mathrm{dL})$, serum albumin $(\mathrm{g} / \mathrm{dL})$, blood urea nitrogen $(\mathrm{BUN}, \mathrm{mg} / \mathrm{dL})$, creatinine $(\mathrm{mg} / \mathrm{dL})$, calcium $(\mathrm{mg} / \mathrm{dL})$, phosphorus $(\mathrm{mg} / \mathrm{dL})$, total cholesterol (mg/dL), high-sensitivity C-reactive protein (hs-CRP, mg/dL), and intact-parathyroid hormone (i-PTH, pg/mL) levels. DM was defined as a self-reported history and medical record of its diagnosis, or a fasting glucose level of $\geq 126 \mathrm{mg} / \mathrm{dL}$. CAD was defined as a self-reported history and medical record of angina, myocardial infarction, or congestive heart failure. CVD was defined as a self-reported history and medical record of stroke.

\section{Assessment of disability and clinical outcomes}

We evaluated disability by using four questions on activities of daily living (ADLs) concerning whether help was needed for feeding, dressing/undressing, getting in/out of bed, or taking a bath/shower [6]. Each question required one of three responses: no help, some help, or full help. We divided the patients into three groups: Non-D, (non-disabled, none of the four ADL domains required help), Mild-D (mildly disabled, one ADL domain required some or full help), or MS-D (moderate to severely disabled, two or more $\mathrm{ADL}$ domains required some or full help).

All data on mortality and hospitalization rates were retrieved from medical records up to March 2014 (mean follow-up duration, $484 \pm 113$ days). If a patient with $\mathrm{HD}$ was admitted for a vascular access-related problem, the hospitalization was not considered as a significant one. We evaluated exhaustion using the vitality (VT) component in HRQoL scales, which consist of four questions: "Did you feel full of pep?", "Did you have a lot of energy?", “Did you feel worn out?", and “Did you feel tired?" Exhaustion was defined as mean vital score $<5$ on four questions [ 5$]$. We defined a fall as an event that resulted in a participant coming to rest unintentionally on the ground or a lower level with or without losing consciousness during the last 1 year. Serious falls were defined as head injury requiring hospitalization, joint dislocations, severe sprains, or a laceration requiring sutures [7]. We identified numbers of participants with fall history and total numbers of falls and/or numbers of serious falls. 
Exercise was classified using the WHO definition [8]. Physical activity was defined as the presence of regular exercise during leisure time for the past 3 months. Exercise was defined as engaging in moderate activity for $>30$ minutes/day for 5 days a week or at a high intensity for $>20$ minutes/day for 3 days a week. Frailty was defined using modified criteria from the study by Johansen et al. [9]. Johansen's criteria include four components (slowness/weakness, 2 points for physical functioning scale $<75$; poor endurance/ exhaustion, 1 point for VT scale $<55$; physical inactivity, 1 point for physically active $<1$ day/week during their leisure time for the past 3 months; and unintentional weight loss, 1 point for unintentional body weight loss $>4.5 \mathrm{~kg}$ or $5 \%$ of baseline value over the past year). A summed score $\geq 3$ is defined as frailty.

\section{Health-related quality of life assessment}

The Kidney Disease Quality of Life-Short Form (KDQOL-SF) Korean version 1.3 was used to evaluate HRQoL [10]. This questionnaire includes the 36-Item Short Form Health Survey (SF-36) with eight domains (physical functioning, role limitations due to physical health problems, body pain, general health, VT, social functioning, role limitations due to emotional problems, and mental health) and an overall health rating. It also includes a kidney disease-specific scale with 11 items (symptom/problems [SP], effects of kidney disease [EKD], burden of kidney disease [BKD], work status [WS], cognitive function [CF], quality of social interaction [QSI], sexual function [SeF], sleep, social support [SS], patient satisfaction [PS], and dialysis staff encouragement [DSE]). Each domain was scored from 0 to 100 . The physical component scale (PCS) and mental component scale (MCS) scores were also calculated from the SF-36 scale [11].

\section{Statistical analyses}

IBM SPSS Statistics version 23 (IBM Corp., Armonk, NY, USA) was used for analyses. Our data were expressed as the mean \pm standard deviation (SD) or standard error (SE) for continuous data and as numbers (percentage) for categorical data. Pearson chisquare test was used to analyze categorical data. One-way analysis of variance, followed by a post-hoc Tukey comparison, was used to analyze continuous data. The Kaplan-Meier log-rank test and Cox regression analysis were used to compare survival estimates. Multivariate methodology was used for the analysis of covariance or Cox regression analysis. The covariates included age, sex, BMI, DM, $\mathrm{CAD}, \mathrm{CVD}$, dialysis vintage, dialysis modality, education level, and levels of hemoglobin, serum albumin, BUN, serum creatinine, calcium, phosphorus, i-PTH, total cholesterol, and hs-CRP. Logistic regression analysis was used to estimate the odds ratios (ORs) and 95\% confidence intervals (CIs), which were then used to deter- mine the correlation between falls and the groups. The level of statistical significance was set at $p<0.05$.

\section{Results}

\section{Clinical characteristics}

The numbers of participants in the Non-D, Mild-D, and MS-D groups were 1,312,163, and 140, respectively (Table 1), and the prevalence of disability was $18.8 \%$ in our cohort. Patients in the MS-D group were the oldest among those in the three groups. The proportion of those with comorbidities (DM, CAD, and CVD) increased as the grade of disability increased. The Non-D group had the highest proportion of those on HD. Serum albumin, creatinine, and phosphorus levels in the MS-D group were lower than those in the Non-D group. The mean follow-up duration was $484 \pm 113$ days. The trend showed lesser follow-up duration in the Mild-D group than in the other groups ( $p=0.046$ for trends; $p=0.037$ for Non-D vs. Mild-D; $p=0.998$ for Non-D vs. MS-D; $p=0.161$ for Mild-D vs. MS-D).

\section{Evaluation of falls, frailty, and exhaustion according to disability}

The numbers of participants who had a fall during the past 1 year were 199 (15.2\%), 42 (25.8\%), and 44 (31.4\%) in the Non-D, Mild-D, and MS-D groups, respectively $(p<0.001)$. In addition, the numbers of participants who had a serious fall during the past 1 year were 35 (2.7\%), 5 (3.1\%), and $6(4.3 \%)$ in the Non-D, Mild-D, and MS-D groups, respectively $(p<0.001)$. The total numbers of falls during the past 1 year were $0.30 \pm 1.51$, $0.42 \pm 0.84$, and $0.57 \pm 1.01$ in the Non-D, Mild-D, and MS-D groups, respectively $(p=0.075)$.

Univariate logistic regression analysis showed that the ORs of falls in the Mild-D and MS-D groups were 1.94 (95\% CI, 1.33$2.85 ; p=0.001)$ and 2.62 (95\% CI, 2.62-3.85; $p<0.001)$, respectively, relative to the Non-D group. The OR of falls in the MS-D group was 1.35 (95\% CI, $0.82-2.22 ; p=0.238$ ), relative to the Mild-D group. Multivariate logistic regression analysis showed that the ORs of falls in the Mild-D and MS-D groups were 1.62 (95\% CI, 0.97-2.70; $p=0.066$ ) and 2.03 (95\% CI, 1.18-3.49; $p=0.011$ ), respectively, relative to the Non-D group. The OR of falls in the MS-D group was 1.38 (95\% CI, 0.68-2.81; $p=0.369$ ), relative to the Mild-D group.

The numbers of participants with frailty in the Non-D, Mild-D, and MS-D groups were 381 (29.0\%), 84 (51.5\%), and 93 (66.4\%), respectively $(p<0.001)$. Additionally, the numbers of participants with exhaustion in the Non-D, Mild-D, and MS-D groups were $844(64.3 \%), 112(68.7 \%)$, and $114(81.4 \%)$, respectively 
Table 1. Clinical characteristics

\begin{tabular}{|c|c|c|c|c|}
\hline Characteristic & Non-D $(n=1,312)$ & Mild-D $(n=163)$ & MS-D $(n=140)$ & $p$-value \\
\hline Age (yr) & $55.0 \pm 12.7$ & $57.9 \pm 13.5$ & $61.1 \pm 13.1^{b, c)}$ & $<0.001$ \\
\hline Male sex & $736(56.1)$ & $92(56.4)$ & 75 (53.2) & 0.795 \\
\hline Body mass index $\left(\mathrm{kg} / \mathrm{m}^{2}\right)$ & $22.4 \pm 3.2$ & $22.3 \pm 3.1$ & $22.4 \pm 3.4$ & 0.967 \\
\hline Diabetes mellitus & 496 (37.8) & $66(40.5)$ & $77(54.6)$ & 0.001 \\
\hline Coronary artery disease & $193(14.7)$ & 25 (15.3) & $36(25.5)$ & 0.004 \\
\hline Cerebrovascular disease & $103(7.9)$ & $19(11.7)$ & $22(15.6)$ & 0.004 \\
\hline Dialysis vintage (yr) & $5.1 \pm 4.4$ & $5.4 \pm 4.5$ & $5.0 \pm 4.9$ & 0.729 \\
\hline Education level & & & & 0.579 \\
\hline$\leq 6$ th grade & $278(21.2)$ & $41(25.2)$ & $34(24.3)$ & \\
\hline 7th-12th grade & 259 (19.7) & $35(21.5)$ & $26(18.6)$ & \\
\hline$>12$ th grade & $775(59.1)$ & $87(53.4)$ & $80(57.1)$ & \\
\hline Hemoglobin (mg/dL) & $10.5 \pm 0.9$ & $10.4 \pm 0.9$ & $10.4 \pm 1.1$ & 0.546 \\
\hline Serum albumin (mg/dL) & $3.9 \pm 0.4$ & $3.8 \pm 0.4$ & $3.8 \pm 0.4^{b)}$ & $<0.001$ \\
\hline BUN (mg/dL) & $60.6 \pm 15.0$ & $57.6 \pm 15.6$ & $57.5 \pm 14.1$ & 0.007 \\
\hline Creatinine (mg/dL) & $10.6 \pm 3.0$ & $10.1 \pm 2.9$ & $9.6 \pm 3.0^{b)}$ & $<0.001$ \\
\hline Calcium (mg/dL) & $8.72 \pm 0.83$ & $8.64 \pm 0.76$ & $8.58 \pm 0.84$ & 0.085 \\
\hline Phosphorus (mg/dL) & $5.37 \pm 1.36$ & $5.06 \pm 1.35$ & $4.79 \pm 1.18^{\mathrm{b}, c)}$ & $<0.001$ \\
\hline Total cholesterol (mg/dL) & $153.5 \pm 36.7$ & $156.1 \pm 38.6$ & $156.2 \pm 40.9$ & 0.551 \\
\hline i-PTH (pg/mL) & $283.8 \pm 345.1$ & $277.5 \pm 378.2$ & $216.8 \pm 254.9$ & 0.087 \\
\hline hs-CRP (mg/dL) & $0.68 \pm 1.63$ & $0.76 \pm 1.93$ & $0.62 \pm 1.29$ & 0.837 \\
\hline Dialysis modality (HD) & $1,053(80.3)$ & $103(63.2)$ & $94(66.7)$ & $<0.001$ \\
\hline Follow-up (day) & $487 \pm 114$ & $463 \pm 125^{b)}$ & $487 \pm 82^{c)}$ & 0.046 \\
\hline
\end{tabular}

Values are presented as mean \pm standard deviation for continuous variables and number (\%) for categorical variables.

Non-D, non-disabled group; Mild-D, mildly disabled group; MS-D, moderate to severely disabled group; BUN, blood urea nitrogen; i-PTH, intactparathyroid hormone; hs-CRP, high-sensitivity C-reactive protein; HD, hemodialysis.

a) $p$-values were tested by using one-way analysis of variance, followed by a post-hoc Tukey comparison, for continuous variables, and Pearson chi-square test for categorical variables. ${ }^{b)} p<0.05$ vs. the Non-D group. ${ }^{c} p<0.05$ vs. the Mild-D group. There was no significant difference in variables between groups without superscripts, such as ${ }^{b)}$ or ${ }^{c}$.

$(p<0.001)$. The numbers of participants who performed exercise in the Non-D, Mild-D, and MS-D groups were 313 (23.9\%), 30 (18.4\%), and $20(14.3 \%)$, respectively $(p=0.023)$.

\section{Evaluation of health-related quality of life scales, hospitalization, and survival according to disability}

Table 2 shows differences in the HRQoL scales according to disability. On both univariate and multivariate analyses, the PCS and MCS scores decreased as the grade of disability increased. Among kidney disease-specific scales, SP, EKD, BKD, WS, CF, QSI, sleep, and PS were associated with the grade of disability on both univariate and multivariate analyses.

Kaplan-Meier analysis showed that those in the Mild-D and MS-D groups had poor first-hospitalization-free survival compared with those in the Non-D group (Fig. 2A). The Non-D group had better patient survival compared with the Mild-D group, but there were no significant differences in survival between the Non-D and MS-D groups or the Mild-D and MS-D groups (Fig. 2B). The total numbers of deaths during follow-up in the Non-D, Mild-D, and
MS-D groups were $59(4.5 \%), 16(9.8 \%)$, and $11(7.9 \%)$, respectively $(p=0.007)$, which was lower in the Non-D group than in the other groups. The most common cause of deaths in the Non-D and Mild-D groups was CAD (15 [25.4\%] in Non-D group and 4 $[25.0 \%]$ in Mild-D group), which that in the MS-D group was infection (5 [45.5\%]). Multivariate Cox regression analysis showed that the Mild-D group had a higher hazard ratio relative to the Non-D group (Table 3). Although statistical significance was not observed, the MS-D group also had a higher hazard ratio relative to the Non-D group. Trends for first-hospitalization-free survival rate were similar to those for patient survival.

\section{Discussion}

The proportion of participants with falls, frailty, and exhaustion increased as the grade of disability increased. Participants with disabilities had low physical activity. Both univariate and multivariate analyses showed an inverse association between the grade of disability and the PCS or MCS. In addition, SP, EKD, BKD, WS, CF, 
Table 2. Comparison of quality-of-life scales according to disability

\begin{tabular}{|c|c|c|c|c|c|c|c|c|}
\hline \multirow{2}{*}{ Scale } & \multicolumn{4}{|c|}{ Univariate (mean \pm SD) } & \multicolumn{4}{|c|}{ Multivariate (mean $\pm \mathrm{SE})$} \\
\hline & Non-D & Mild-D & MS-D & $p$-value & Non-D & Mild-D & MS-D & $p$-value \\
\hline \multicolumn{9}{|c|}{ SF-36 scale } \\
\hline $\mathrm{PF}$ & $78.4 \pm 20.0$ & $62.2 \pm 26.3^{\mathrm{a})}$ & $49.2 \pm 31.9^{\mathrm{a}, \mathrm{b})}$ & $<0.001$ & $76.6 \pm 0.8$ & $64.4 \pm 1.8^{\mathrm{a})}$ & $51.4 \pm 2.1^{\mathrm{a}, \mathrm{b})}$ & $<0.001$ \\
\hline $\mathrm{RP}$ & $69.5 \pm 39.6$ & $49.3 \pm 41.8^{\mathrm{a})}$ & $38.7 \pm 43.9^{\mathrm{a}, \mathrm{b})}$ & $<0.001$ & $69.9 \pm 1.5$ & $55.4 \pm 3.7^{\text {a) }}$ & $35.4 \pm 4.2^{\mathrm{a}, \mathrm{b})}$ & $<0.001$ \\
\hline $\mathrm{BP}$ & $80.1 \pm 24.1$ & $70.5 \pm 27.3^{\mathrm{a})}$ & $61.4 \pm 28.2^{\mathrm{a}, \mathrm{b})}$ & $<0.001$ & $79.8 \pm 0.9$ & $74.2 \pm 2.3^{\mathrm{a})}$ & $59.7 \pm 2.6^{a, b)}$ & $<0.001$ \\
\hline $\mathrm{GH}$ & $46.0 \pm 22.3$ & $39.2 \pm 21.9^{a)}$ & $35.1 \pm 21.9^{a)}$ & $<0.001$ & $46.0 \pm 0.9$ & $40.8 \pm 2.1^{\mathrm{a})}$ & $33.3 \pm 2.4^{\mathrm{a})}$ & $<0.001$ \\
\hline $\mathrm{VT}$ & $46.5 \pm 20.8$ & $39.4 \pm 20.6^{a)}$ & $34.6 \pm 22.2^{\mathrm{a}, \mathrm{b})}$ & $<0.001$ & $46.6 \pm 0.8$ & $41.6 \pm 2.0^{a)}$ & $34.7 \pm 2.2^{\mathrm{a})}$ & $<0.001$ \\
\hline SF & $78.1 \pm 26.7$ & $66.6 \pm 27.8^{\mathrm{a})}$ & $60.9 \pm 31.6^{a)}$ & $<0.001$ & $77.9 \pm 1.1$ & $67.8 \pm 2.6^{a)}$ & $59.6 \pm 3.0^{\mathrm{a})}$ & $<0.001$ \\
\hline $\mathrm{RE}$ & $75.1 \pm 39.6$ & $55.3 \pm 44.6^{a)}$ & $49.2 \pm 45.9^{\mathrm{a})}$ & $<0.001$ & $76.6 \pm 1.6$ & $63.2 \pm 3.8^{\mathrm{a})}$ & $41.9 \pm 4.3^{\mathrm{a}, \mathrm{b})}$ & $<0.001$ \\
\hline $\mathrm{MH}$ & $60.8 \pm 19.7$ & $52.7 \pm 20.0^{a)}$ & $48.3 \pm 22.7^{\mathrm{a})}$ & $<0.001$ & $62.0 \pm 0.8$ & $55.0 \pm 1.9^{a)}$ & $46.7 \pm 2.2^{\mathrm{a}, \mathrm{b})}$ & $<0.001$ \\
\hline $\mathrm{OHR}$ & $39.0 \pm 25.4$ & $33.7 \pm 28.9$ & $27.8 \pm 25.7^{\mathrm{a}, \mathrm{b})}$ & $<0.001$ & $37.4 \pm 1.0$ & $34.9 \pm 2.4$ & $27.4 \pm 2.7^{\mathrm{a}, \mathrm{b})}$ & 0.003 \\
\hline PCS & $64.1 \pm 18.9$ & $52.1 \pm 21.1^{\text {a) }}$ & $43.8 \pm 23.2^{a, b)}$ & $<0.001$ & $63.8 \pm 0.7$ & $55.3 \pm 1.8^{\mathrm{a})}$ & $42.9 \pm 2.0^{\mathrm{a}, \mathrm{b})}$ & $<0.001$ \\
\hline MCS & $61.3 \pm 19.5$ & $50.7 \pm 20.3^{\mathrm{a})}$ & $45.6 \pm 22.5^{\mathrm{a}, \mathrm{b})}$ & $<0.001$ & $61.8 \pm 0.8$ & $53.7 \pm 1.8^{\mathrm{a})}$ & $43.2 \pm 2.1^{a, b)}$ & $<0.001$ \\
\hline \multicolumn{9}{|c|}{ KD-specific scale } \\
\hline SP & $81.9 \pm 13.5$ & $74.5 \pm 17.1^{a)}$ & $70.6 \pm 18.9^{\mathrm{a}, \mathrm{b})}$ & $<0.001$ & $82.4 \pm 0.6$ & $77.3 \pm 1.3^{\mathrm{a})}$ & $69.3 \pm 1.5^{\mathrm{a}, \mathrm{b})}$ & $<0.001$ \\
\hline EKD & $75.2 \pm 17.9$ & $66.3 \pm 20.8^{\mathrm{a})}$ & $60.6 \pm 22.3^{a, b)}$ & $<0.001$ & $76.6 \pm 0.7$ & $67.6 \pm 1.7^{\mathrm{a})}$ & $57.6 \pm 1.9^{\mathrm{a}, \mathrm{b})}$ & $<0.001$ \\
\hline BKD & $36.8 \pm 26.2$ & $27.4 \pm 25.3^{\mathrm{a})}$ & $27.7 \pm 26.6^{a)}$ & $<0.001$ & $37.2 \pm 1.0$ & $27.3 \pm 2.4^{\mathrm{a})}$ & $25.2 \pm 2.8^{\mathrm{a})}$ & $<0.001$ \\
\hline WS & $29.9 \pm 37.1$ & $21.2 \pm 32.3^{\mathrm{a})}$ & $16.7 \pm 29.1^{a)}$ & $<0.001$ & $29.3 \pm 1.3$ & $21.3 \pm 3.3^{\mathrm{a})}$ & $23.9 \pm 3.8$ & 0.049 \\
\hline $\mathrm{CF}$ & $88.1 \pm 15.2$ & $80.3 \pm 20.9^{a)}$ & $73.2 \pm 24.3^{\mathrm{a}, \mathrm{b})}$ & $<0.001$ & $88.9 \pm 0.6$ & $82.7 \pm 1.5^{\mathrm{a})}$ & $70.7 \pm 1.8^{a, b)}$ & $<0.001$ \\
\hline OSI & $77.5 \pm 20.6$ & $69.2 \pm 21.9^{\mathrm{a})}$ & $62.7 \pm 26.3^{\mathrm{a}, \mathrm{b})}$ & $<0.001$ & $77.0 \pm 0.8$ & $69.8 \pm 2.0^{\text {a) }}$ & $58.6 \pm 2.3^{a, b)}$ & $<0.001$ \\
\hline $\mathrm{SeF}$ & $78.1 \pm 25.9$ & $69.3 \pm 29.9$ & $77.2 \pm 24.0$ & 0.230 & $77.7 \pm 2.2$ & $69.0 \pm 6.1$ & $88.5 \pm 8.3$ & 0.155 \\
\hline Sleep & $65.6 \pm 21.0$ & $57.4 \pm 21.6^{\mathrm{a})}$ & $56.7 \pm 21.0^{a)}$ & $<0.001$ & $66.7 \pm 0.8$ & $56.8 \pm 1.9^{a)}$ & $55.6 \pm 2.2^{\mathrm{a})}$ & $<0.001$ \\
\hline SS & $68.9 \pm 26.9$ & $59.2 \pm 27.2^{\mathrm{a})}$ & $66.7 \pm 26.3^{b)}$ & $<0.001$ & $67.6 \pm 1.1$ & $61.5 \pm 2.6$ & $65.5 \pm 2.9$ & 0.084 \\
\hline PS & $66.6 \pm 22.7$ & $61.7 \pm 24.4$ & $63.1 \pm 21.5$ & 0.013 & $66.5 \pm 0.9$ & $60.3 \pm 2.1$ & $63.2 \pm 2.4$ & 0.019 \\
\hline DSE & $86.2 \pm 18.1$ & $84.7 \pm 20.5$ & $87.9 \pm 18.5$ & 0.310 & $88.3 \pm 0.7$ & $85.6 \pm 1.6$ & $86.6 \pm 1.8$ & 0.260 \\
\hline
\end{tabular}

$p$-values were determined by using Student's t-test for univariate analysis and analysis of covariance for multivariate analysis. The multivariate analysis was adjusted for age, sex, body mass index, dialysis modality, diabetes mellitus, cerebrovascular disease, coronary artery disease, dialysis vintage, education level, levels of hemoglobin, serum albumin, blood urea nitrogen, serum creatinine, calcium, phosphorus, total cholesterol, intact-parathyroid hormone, and high-sensitivity C-reactive protein.

SD, standard deviation; SE, standard error; Non-D, non-disabled group; Mild-D, mildly disabled group; MS-D, moderate to severely disabled group; SF-36, 36-Item Short Form Health Survey; PF, physical functioning; RP, role limitations due to physical health problems; BP, body pain; GH, general health; VT, vitality; SF, social functioning; RE, role limitations due to emotional problems; $M H$, mental health; OHR, overall health rating; PCS, physical component scale; MCS, mental component scale; KD, kidney disease; SP, symptom/problems; EKD, effects of kidney disease; BKD, burden of kidney disease; WS, work status; CF, cognitive function; QSI, quality of social interaction; SeF, sexual function; SS, social support; PS, patient satisfaction; DSE, dialysis staff encouragement.

${ }^{a)} p<0.05$ vs. the Non-D group. ${ }^{\text {b) }} p<0.05$ vs. the Mild-D group. There was no significant difference in variables between groups without superscripts, such as ${ }^{\text {a) }}$ or $^{\text {b) }}$.

QSI, sleep, and PS were associated with the grade of disability in both univariate and multivariate analyses. Although the statistical significance was weak, the trend was favorable for the first-hospitalization-free and patient survival rates in the Non-D group. However, there were no significant differences in these rates between the Mild-D and MS-D groups.

Disability was defined as an umbrella term for impairments, activity limitations, and participation restrictions [2]. Social, medical, or psychological problems lead to functional or intellectual disability, which is associated with inaccessibility to health care services or vulnerability to diseases such as sore or urinary tract infection. Disability can be diagnosed using a medical, functional, or social mod- el, with the functional model (using ADL) being the most commonly used one [12]. Katz et al. [13] first published a model using $6 \mathrm{ADLs}$ (bathing, dressing, toileting, transfer, continence, or feeding). Modified scales using 4 or $5 \mathrm{ADLs}$ were introduced after the original version $[6,14,15]$. We evaluated disability using a model with 4 ADLs [6].

Dialysis patients are at a high risk of falls and these are associated with adverse outcomes. In addition, previous studies have shown an association between frailty/exhaustion and cardiovascular disease or mortality, in dialysis and nondialysis populations $[16,17]$. Therefore, we aimed to identify the association between disability and these variables. First, we analyzed cross-sectional data at en- 

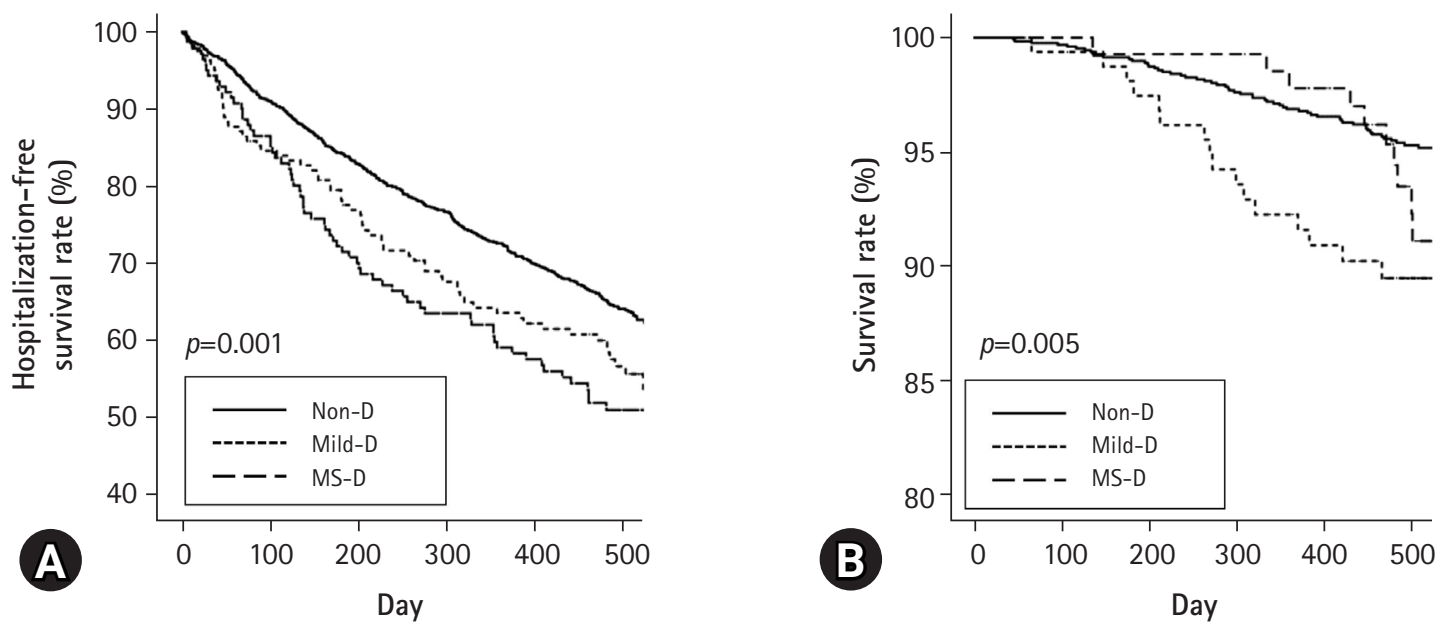

Fig. 2. Kaplan-Meier curves of hospitalization-free survival and patient survival. (A) Hospitalization-free survival rate at 500 days (Non-D, 64.2\%; Mild-D, 56.7\%; MS-D, 51.1\%; $p=0.001$ for trends, $p=0.023$ for Non-D vs. Mild-D, $p=0.001$ for Non-D vs. MS-D, and $p=0.445$ for Mild-D vs. MS-D). (B) Patient survival rate at 500 days (Non-D, 95.3\%; Mild-D, 89.5\%; MS-D, 92.3\%; $p=0.005$ for trends, $p=0.003$ for Non-D vs. Mild-D, $p=0.088$ for Non-D vs. MS-D, and $p=0.434$ for Mild-D vs. MS-D). Non-D, non-disabled group; Mild-D, mildly disabled group; MS-D, moderate to severely disabled group.

Table 3. Cox regression analyses according to disability

\begin{tabular}{|c|c|c|c|c|}
\hline \multirow{2}{*}{ Variable } & \multicolumn{2}{|c|}{ Univariate } & \multicolumn{2}{|c|}{ Multivariate } \\
\hline & Hazard ratio $(95 \% \mathrm{Cl})$ & $p$-value & Hazard ratio $(95 \% \mathrm{Cl})$ & $p$-value \\
\hline \multicolumn{5}{|l|}{ Patient survival } \\
\hline Mild-D & $2.32(1.34-4.03)$ & 0.003 & $2.15(1.08-4.30)$ & 0.030 \\
\hline MS-D & $1.73(0.91-3.29)$ & 0.095 & $1.26(0.55-2.87)$ & 0.583 \\
\hline Mild-D (ref) & - & - & - & - \\
\hline \multicolumn{5}{|l|}{ First HFS } \\
\hline Non-D (ref) & - & - & - & - \\
\hline Mild-D & $1.34(1.04-1.72)$ & 0.025 & $1.33(0.98-1.81)$ & 0.064 \\
\hline MS-D & $1.52(1.18-1.97)$ & 0.001 & $1.23(0.89-1.71)$ & 0.210 \\
\hline Mild-D (ref) & - & - & - & - \\
\hline
\end{tabular}

Multivariate analysis was adjusted for age, sex, body mass index, dialysis modality, diabetes mellitus, cerebrovascular disease, coronary artery disease, dialysis vintage, education level, and levels of hemoglobin, serum albumin, blood urea nitrogen, serum creatinine, calcium, phosphorus, total cholesterol, intact-parathyroid hormone, and high-sensitivity C-reactive protein.

$\mathrm{Cl}$, confidence interval; Non-D, non-disabled group; Mild-D, mildly disabled group; MS-D, moderate to severely disabled group; ref, reference; HFS, hospitalization-free survival.

rollment and found that the presence of disability was associated with falls. Although a causal relationship between the two variables was not clear, we suggested that patients with disabilities would be at risk of falls and those who have had falls would be prone to having disabilities. Second, our study showed an association between disability and frailty/exhaustion. Frailty is defined as a medical syndrome with multiple etiologies that is associated with decreased strength, endurance, and physical function [17]. Sense of exhaustion is defined as a combination of fatigue, lack of energy, feelings of hopelessness, loss of libido, or increased irritability [16]. In our study, frailty was evaluated based on physical function/performance, VT, and unintentional weight loss. Exhaustion was evaluated using VT component in HRQoL scales. Therefore, frailty focused on physical function and exhaustion focused on subjective sense. Although the two variables closely overlap or correlate, they are different. Our study showed that disability was associated with these two variables (frailty and exhaustion).

Disability was associated with falls, frailty, and exhaustion, all of 
which can in turn lead to disability; exercise or rehabilitation may be helpful to prevent or decrease these vicious cycles. El-Khoury et al. [18] performed a meta-analysis for the effect of the exercise program on fall-induced injuries in an elderly population and showed that exercise results in a decrease in all injurious falls. Desai et al. [19] evaluated the favorable effect of pedaling exercise on a 6-minute walking test and handgrip and pinch strengths in $\mathrm{HD}$ patients. A Korean study evaluated the positive effect of intradialytic exercise on daily physical activity, and it was found to improve physical activity [20]. Additionally, Lorenz et al. [21] showed a favorable result of a protocolized exercise program in kidney transplant candidates with advanced chronic kidney disease. However, our results showed that exercise decreased as the grade of disability increased. Education and encouragement of active exercise or rehabilitation would lead to improvement and prevention of adverse outcomes in participants with disabilities or those prone to having disabilities.

We evaluated the effect of disability on HRQoL scales in dialysis patients. Jassal et al. [3] investigated the association between disability scores and HRQoL. However, they evaluated HRQoL using SF-36 alone and only focused on the PCS and MCS among data for the SF-36 scales. In addition, they performed multivariate analyses adjusted by age and country alone. We evaluated HRQLL using the KDQOL-SF and examined the scores for each of the 22 components, including the PCS and MCS. Various covariates were adjusted in our study. Our results showed that all 11 scores in SF36 including the PCS and MCS were associated with disability, which is consistent with previous study findings. Among kidney disease-specific scales, SP, EKD, BKD, WS, CF, QSI, and sleep were associated with disability. However, SeF, SS, PS, and DSE were not associated with disability; among these, SS, PS, and DSE would be more influenced by social factors such as quality of health care or medical insurance than by medical or functional status in each participant.

A prospective study showed the positive association between the presence of disability and mortality in dialysis patients [22]. A multicenter study using the Dialysis Outcomes and Practice Pattern Study cohort enrolled 7,226 HD patients and showed that mortality increased and HRQoL decreased as functional dependency increased [3]. A Japanese study divided the 7,664 HD patients into three groups according to the severity of disability, and a positive association between the grade of disability and early death or all-cause death was observed [4]. In addition, previous studies showed that multi-domain interventions including nutritional, physical, or cognitive treatment were effective in improving physical or emotional function in the elderly population $[23,24]$.

Our results were similar to those from previous studies. This study showed that the Mild-D group had poor patient survival compared with the Non-D group. The MS-D group had poor patient survival compared with the Non-D group, but there was no statistical significance. Additionally, there was no significant difference in patient survival rates between the Mild-D and MS-D groups. The first hospitalization-free survival trend was similar to that of patient survival. The Mild-D group had the poorest survival, possibly because of two factors. First, the use of a subjective method using questionnaires and the lack of consensus for the assessment of the severity of disability are associated with a nonlinear trend between the severity of disability and prognosis. This study did not show poor prognosis in the MS-D group compared to the Mild-D group, and this may be caused by the two aforementioned factors. The presence of disability is associated with high morbidity and mortality. Some studies have shown the association between the severity of disability and prognosis, but there were differences in the methods defining disability and cut-off value for severity $[25,26]$. In addition, Son et al. [25] showed that the presence of disability was associated with poor prognosis, but prognosis did not differ between mild and severe disability. In our study, disability was defined using questionnaires for $\mathrm{ADL}$, which is a highly subjective method. The patients in the MS-D group had significantly lower scores for body pain, MCS, SP, and QSI than those in the Mild-D group. These revealed that the severity of disability can lead to low HRQoL scores, but measuring the severity of disability using a subjective method can be influenced by low HRQoL scores. Second, the short-term follow-up duration and high patient survival rates in our study may not achieve statistical significance. In addition, the numbers of deaths by $\mathrm{CAD}$, infection, or others, respectively, were 15, 13, and 31 in the Non-D group, four, three, and nine in the Mild-D group, and none, five, and six in the MS-D group. The absolute number of deaths was different among the three groups, but there was no statistically significant difference in the distribution of the cause of death among them $(p=0.271)$. Further investigations, using a method combined with objective indicators such as handgrip strength or gait speed and data with greater number of deaths during long-term follow-up, would be helpful to identify the difference in prognosis and cause of death according to the severity of disability.

Our study included both HD and PD patients and showed that the number of $\mathrm{HD}$ patients decreased as the grade of disability increased. Differences in disability and HRQoL according to modality are additional important issues. There were conflicting results regarding the association between disability/HRQoL and dialysis modality. Although our study did not include these data, a previous study using this cohort evaluated the impact of disability by dialysis modality [27]. The study showed that disability was more 
common in PD patients; additionally, MCS and PCS were higher in $\mathrm{HD}$ patients than in $\mathrm{PD}$ patients.

Malnutrition is associated with a loss of muscle mass, which can lead to disability. Serum albumin and creatinine are well-known indicators of nutritional or muscle mass status, and serum phosphorus is highly associated with protein intake. Therefore, malnourished patients with low serum albumin, creatinine, or phosphorus levels are prone to the development of disability. In our study, the Non-D group had trend for higher serum albumin, creatinine, and phosphorus than the other groups. The trend in these variables would be an extension of the high prevalence of malnutrition in patients with disabilities.

This study has a few limitations. First, our study design was retrospective, and we used a data set from a previous study [5]. Second, we did not evaluate disability by using questionnaires with traditional measurements, such as those used by Katz et al. [15]. Questionnaires for five ADLs are most commonly used to define disability [28]. However, our study evaluated disability using four items (meal, dress/undress, get in or out of bed, and take a bath or shower) among the five to six traditional items. Validity of the use of four items has not been evaluated. However, due to limited effort and time commitments, two similar questions such as those for toileting or bathing can be combined into one question. Third, our study did not include data on dialysis adequacy. Fourth, there was a large difference in the numbers among the groups. The numbers in the Non-D group were greater than those in the other groups. The difference in numbers among the groups is common in studies using diseases with low prevalence and this is associated with selection bias. Studies for two groups may be overcome using statistical methods, such as propensity score matching. However, there was little evidence for propensity score matching methods in studies of three or more groups. Future prospective studies with long-term follow-up and additional parameters such as dialysis adequacy are needed to overcome these limitations.

In conclusion, disability was associated with poorer outcomes, which include falls, frailty, and exhaustion in Korean dialysis patients. Most HRQoL scales and survival trends were poorer in participants with disabilities than those without a disability.

\section{Acknowledgments}

\section{Conflicts of interest}

No potential conflict of interest relevant to this article was reported.

\section{Funding}

This study was supported by the National Research Foundation of
Korea (NRF) grant funded by the Korea government (Ministry of Science and ICT) (2017R1C1B5077075), and the Medical Research Center Program (2015R1A5A2009124) through the National Research Foundation of Korea (NRF) funded by the Ministry of Science, ICT and Future Planning. The founder had no role in study design, collection, analysis, and interpretation of data, writing the report, and the decision to submit the report for publication.

\section{Author contributions}

Conceptualization and Funding acquisition: SHK; Data acquisition, SHK, JYD, JCK; Formal analysis and Data interpretation: SHK, JCK; Writing-original draft: SHK, JCK; Writing-review \& editing: all authors.

\section{ORCID}

Seok Hui Kang, https://orcid.org/0000-0003-1023-0195

Jun Young Do, https://orcid.org/0000-0002-6360-9310

Jun Chul Kim, https://orcid.org/0000-0002-7578-6322

\section{References}

1. United States Renal Data System. 2017 USRDS annual data report: epidemiology of kidney disease in the United States. Bethesda (MD): National Institutes of Health, National Institute of Diabetes and Digestive and Kidney Diseases; 2017.

2. World Health Organization. Disability and health [Internet]. Geneva: World Health Organization; 2018 [cited 2017 May 15]. http://www.who.int/mediacentre/factsheets/fs352/en/.

3. Jassal SV, Karaboyas A, Comment LA, Bieber BA, Morgenstern $\mathrm{H}, \mathrm{Sen} \mathrm{A}$, et al. Functional dependence and mortality in the international Dialysis Outcomes and Practice Patterns Study (DOPPS). Am J Kidney Dis 2016;67:283-92.

4. Yazawa M, Kido R, Ohira S, Hasegawa T, Hanafusa N, Iseki K, et al. Early mortality was highly and strongly associated with functional status in incident Japanese hemodialysis patients: a cohort study of the large national dialysis registry. PLoS One 2016;11:e0156951.

5. Lee SY, Yang DH, Hwang E, Kang SH, Park SH, Kim TW, et al. The prevalence, association, and clinical outcomes of frailty in maintenance dialysis patients.J Ren Nutr 2017;27:106-12.

6. Woods NF, LaCroix AZ, Gray SL, Aragaki A, Cochrane BB, Brunner RL, et al. Frailty: emergence and consequences in women aged 65 and older in the Women's Health Initiative Observational Study.J Am Geriatr Soc 2005;53:1321-30.

7. Buchner DM, Hornbrook MC, Kutner NG, Tinetti ME, Ory MG, Mulrow CD, et al. Development of the common data base 
for the FICSIT trials. J Am Geriatr Soc 1993;41:297-308.

8. World Health Organization. Physical activity [Internet]. Geneva: World Health Organization; 2018 [cited 2017 May 15]. http://www.who.int/mediacentre/factsheets/fs385/en/.

9. Johansen KL, Chertow GM, Jin C, Kutner NG. Significance of frailty among dialysis patients. J Am Soc Nephrol 2007;18: 2960-7.

10. Park HJ, Kim S, Yong JS, Han SS, Yang DH, Meguro M, et al. Reliability and validity of the Korean version of Kidney Disease Quality of Life instrument (KDQOL-SF). Tohoku J Exp Med 2007;211:321-9.

11. Ware JE Jr, Sherbourne CD. The MOS 36-item short-form health survey (SF-36). I. Conceptual framework and item selection. Med Care 1992;30:473-83.

12. McDermott S, Turk MA. The myth and reality of disability prevalence: measuring disability for research and service. Disabil Health J 2011;4:1-5.

13. Katz S, Ford AB, Moskowitz RW, Jackson BA, Jaffe MW. Studies of illness in the aged. The index of ADL: a standardized measure of biological and psychosocial function. JAMA 1963;185: 914-9.

14. LaPlante MP. The classic measure of disability in activities of daily living is biased by age but an expanded IADL/ADL measure is not. J Gerontol B Psychol Sci Soc Sci 2010;65:720-32.

15. Katz S, Downs TD, Cash HR, Grotz RC. Progress in development of the index of ADL. Gerontologist 1970;10:20-30.

16. Cole SR, Kawachi I, Sesso HD, Paffenbarger RS, Lee IM. Sense of exhaustion and coronary heart disease among college alumni. Am J Cardiol 1999;84:1401-5.

17. Sy J, Johansen KL. The impact of frailty on outcomes in dialysis. Curr Opin Nephrol Hypertens 2017;26:537-42.

18. El-Khoury F, Cassou B, Charles MA, Dargent-Molina P. The effect of fall prevention exercise programmes on fall induced injuries in community dwelling older adults: systematic review and meta-analysis of randomised controlled trials. BMJ 2013;347: f6234.

19. Desai M, Mohamed A, Davenport A. A pilot study investigating the effect of pedalling exercise during dialysis on 6-min walking test and hand grip and pinch strength. Int J Artif Organs 2019;42:161-6.

20. Cho JH, Lee JY, Lee S, Park H, Choi SW, Kim JC. Effect of intradialytic exercise on daily physical activity and sleep quality in maintenance hemodialysis patients. Int Urol Nephrol 2018;50: 745-54.

21. Lorenz EC, Hickson LJ, Weatherly RM, Thompson KL, Walker HA, Rasmussen JM, et al. Protocolized exercise improves frailty parameters and lower extremity impairment: a promising prehabilitation strategy for kidney transplant candidates. Clin Transplant 2020;34:e14017.

22. McAdams-Demarco MA, Law A, Garonzik-Wang JM, Gimenez L, Jaar BG, Walston JD, et al. Activity of daily living disability and dialysis mortality: better prediction using metrics of aging.J Am Geriatr Soc 2012;60:1981-2.

23. Ng TP, Feng L, Nyunt MS, Feng L, Niti M, Tan BY, et al. Nutritional, physical, cognitive, and combination interventions and frailty reversal among older adults: a randomized controlled trial. Am J Med 2015;128:1225-36.

24. Ng TP, Nyunt MS, Feng L, Feng L, Niti M, Tan BY, et al. Multi-domains lifestyle interventions reduces depressive symptoms among frail and pre-frail older persons: randomized controlled trial. J Nutr Health Aging 2017;21:918-26.

25. Son KY, Kim SH, Sunwoo S, Lee JY, Lim S, Kim YS. Association between disability and cardiovascular event and mortality: a nationwide representative longitudinal study in Korea. PLoS One 2020;15:e0236665.

26. Park JM, Oh U, Roh BR, Moon Y. Disparities in mortality by disability: an 11-year follow-up study of 1 million individuals. Int J Public Health 2017;62:989-96.

27. Kang SH, Do JY, Lee SY, Kim JC. Effect of dialysis modality on frailty phenotype, disability, and health-related quality of life in maintenance dialysis patients. PLoS One 2017;12:e0176814.

28. Tufan F, Topcu Y, Bahat G, Kara MA. Limitations of the continence item in Katz Activities of Daily Living scale. Gerontol Geriatr Res 2016;2:1008. 